\title{
A geometric model analysis of conductive airways in expiratory flow limitation during artificial ventilation
}

\author{
G. Cevenini, F. Bernardi, M.R. Massai, P. Barbini \\ Department of Surgery and Bioengineering, Siena University, Siena, Italy
}

\begin{abstract}
Expiratory flow limitation (EFL) was simulated in mechanical ventilation through a recently proposed tracheobronchial tree model which reproduces breathing mechanics in the time-domain using Weibel's symmetrical description of lung anatomy considered as 17 upper generations conducting air and seven deeper generations where gas exchange takes place. The model allows for flow turbulence, inertance and tissue viscoelasticity to be represented nonlinearly and distributed along the tracheobronchial tree in a lumped parameter description. An electric analogue of the simulator, consisting of a RLC ladder network, was numerically implemented by Matlab-Simulink software. Normal conditions and chronic obstructive pulmonary disease (COPD) were simulated by setting model parameters. An extra negative expiratory pressure (NEP) was introduced to detect EFL. The transmural pressures of conductive airways, their viscoelastic characteristics and their related distributions of transversal geometrical changes over the breathing cycle were evaluated. The results showed that during mechanical ventilation, large modifications in the elastic characteristics of the conductive airways explain EFL in simulated COPD. In particular, a marked expiratory reduction in medium bronchi diameter, up to $20-30 \%$ of their maximum value, and a progressive decrease in small bronchi diameter during expiration were observed. A remarkably lower reduction in medium bronchi diameter and almost constant behaviour of small bronchi diameter during expiration were found in the simulated healthy subject. Application of NEP confirmed the absence of EFL for normal cases whereas COPD was flow limited over most of expiration.
\end{abstract}




\section{Introduction}

Expiratory flow limitation (EFL) is an important effect of breathing mechanics caused by pathophysiological changes of airway viscoelastic properties in many cases due to chronic obstructive pulmonary diseases (COPD) [1,2]. In mechanically ventilated COPD patients, therapy and ventilation strategy depend critically on severity and may be optimised by knowledge of the degree of EFL.

Standard well-known techniques based on flow-volume curves, such as negative expiratory pressure (NEP), can be used to quantify patient EFL with good accuracy [3]. However, these techniques are not designed to evaluate the distribution of transmural pressure along the tracheobronchial tree; this distribution produces geometric modifications due to airway and tissue viscoelasticity. A morphometry-based analysis of the pathophysiological mechanisms that produce EFL could be a useful auxiliary in the management of critical patients.

Here we attempt to interpret the pathophysiological mechanical changes in the tracheobronchial tree underlying EFL in artificial ventilation. We use a morphometric model recently proposed by Bernardi et al. [4], derived from the symmetrical lung description of Weibel [5] appearing in many respiratory physiology textbooks. Although the anatomical conformation of the tracheobronchial tree is asymmetrical, the main system characteristics are readily explained by this symmetrical model which is a good compromise between complexity and descriptive detail. In a previous study by Cevenini et al. [6], the model was demonstrated to be particularly suited for analysing changes in conductive-airway transversal sections during expiration.

Here we study EFL, setting model parameters by means of a computer simulation approach.

\section{Methods}

\subsection{Morphometric model of the tracheobronchial tree}

The morphometric nonlinear model used to describe breathing mechanics in artificial ventilation is based on the Weibel [5] description of tracheobronchial tree geometry. Weibel divided the lung into 24 generations. The trachea was identified as generation zero and the alveolar sacs as generation 23. Figure 1 shows anatomical details of the Weibel model. The first 17 and the last seven generations constitute the conductive and respiratory zones, respectively. Our model organises conductive airways symmetrically into 17 generations, each of which bifurcates from the trachea to the smaller bronchioles, while the respiratory zone is considered as a single compartment. Conductive airways are modelled as compliant cylinders, the diameter of which varies nonlinearly with transmural pressure, accounting for flow turbulence, inertance and viscoelasticity.

Since the wavelengths of breathing signals are much greater than anatomical 
lung size, the distributed nature of resistance, compliance and inertance can be neglected and each conductive branch can be represented electrically as a lumped-parameter RLC cell [4,6]. Figure 2 shows the electrical analogue of bronchial branching. By virtue of their symmetrical organisation, the conductive airways can be represented electrically as a ladder network of 17 RLC cells, one for each generation.

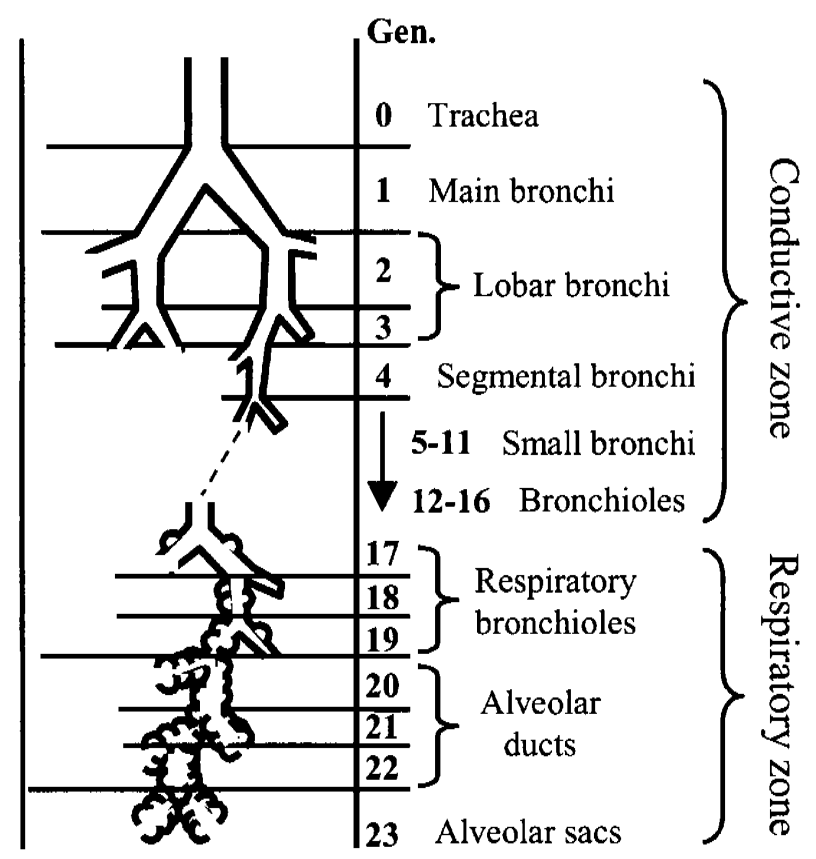

Figure 1: Weibel morphometric model of the lungs.

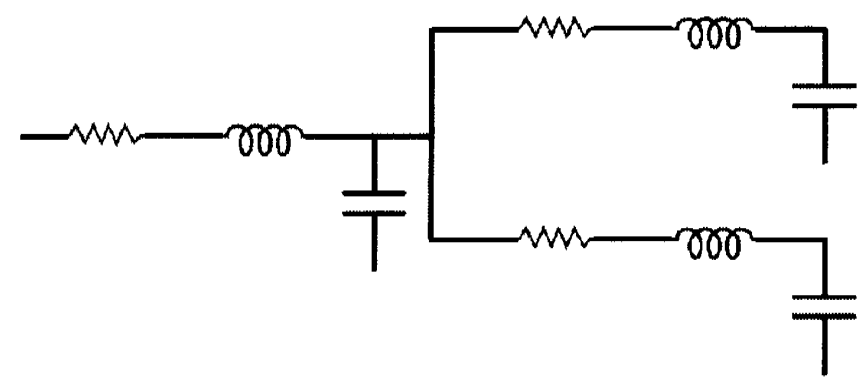

Figure 2: Electric analogue of bronchial branching of the morphometric model. A parent branch give rises to two identical daughter branches. 
The respiratory zone was modelled by a $\mathrm{RC}$ cell, taking chest wall compliance into account.

In the conducting zone, total resistance, $R_{n}$, inertance, $L_{n}$, and compliance, $C_{n}$, of generation $n$ were obtained by the parallel of $2^{n}$ branches, [6]:

$$
\begin{aligned}
& R_{n}=\frac{1}{2^{n}} \cdot \frac{128 \cdot \eta \cdot l_{n}^{(\mathrm{b})}}{\pi \cdot\left(d_{n}^{(\mathrm{b})}\right)^{4}}\left(1+k_{n}\left|\dot{V}_{n}\right|\right) \\
& L_{n}=\frac{1}{2^{n}} \cdot \frac{4 \cdot \rho \cdot l_{n}^{(\mathrm{b})}}{\pi \cdot\left(d_{n}^{(\mathrm{b})}\right)^{2}} \\
& C_{n}=\frac{1}{E_{n}}=2^{n} \cdot \frac{1}{\frac{\mathrm{d} P_{n}^{(\mathrm{b})}}{\mathrm{d} V_{n}^{(\mathrm{b})}}} \\
& P_{n}^{(\mathrm{b})}=\frac{A_{1 n}}{d_{n \max }^{(\mathrm{b})}-d_{n}^{(\mathrm{b})}}+\frac{A_{2 n}}{d_{n \text { min }}^{(\mathrm{b})}-d_{n}^{(\mathrm{b})}}+A_{3 n} \\
& d_{n}^{(\mathrm{b})}=2 \cdot \sqrt{\frac{V_{n}^{(\mathrm{b})}}{\pi \cdot l_{n}^{(\mathrm{b})}}}
\end{aligned}
$$

Resistance $R_{n}$ was described by a Rohrer representation which accounted for turbulent flow by means of the constant, $k_{n}$, estimated from experimental data, and the generation flow, $\dot{V}_{n}$. The first addendum of eqn (1) is the Poiseuille laminar resistance where $\eta$ is air viscosity, $l_{n}^{(\mathrm{b})}$ the length and $d_{n}^{(\mathrm{b})}$ the diameter of a single branch (b).

Inertance $L_{n}$ in eqn (2) typically depends on air density, $\rho$, and branch length and diameter.

For convenience, compliance $C_{n}$ in eqn (3) is defined through its inverse, elastance, $E_{n}$. In fact $E_{n}$ is easily obtained from eqns (4) and (5) by the derivative of branch transmural pressure, $P_{n}^{(b)}$, with respect to branch volume, $V_{n}^{(\text {b) }}$. Eqn (4) defines mathematically a sigmoid elastic characteristic of the wall [7]; $d_{n \max }^{(\mathrm{b})}$ and $d_{n \min }^{(\mathrm{b})}$ are the maximum and minimum branch diameter; $A_{1_{n}}$, $A_{2 n}$ and $A_{3 n}$ are important model parameters, the values of which determine the shape of the sigmoid and therefore the elastic properties of the generation. In particular, referring to Fig. 3, where the branch diameter is reported as a 
percentage of its maximum value, $A_{1 n}$ and $A_{2 n}$ determine the curvature of upper-right and lower-left parts, respectively and also of course influence the central slope of curve. $A_{3_{n}}$ represents horizontal curve shift.

The respiratory zone is represented as a single RC cell with constant values, while chest wall is modelled as a constant elastance [8].

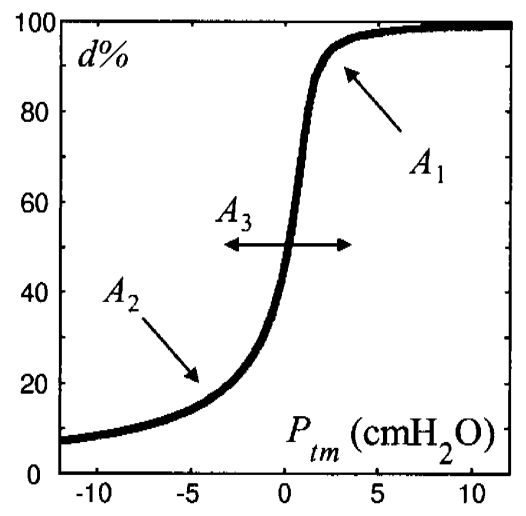

Figure 3: Modelling of elastic characteristic of conductive airways: branch diameter percentage, $d \%$, as function of the transmural pressure, $P_{t m}$.

\subsection{Mechanical ventilator}

A respiratory frequency of 15 breaths/minute was assumed. As outlined by Barbini et al. [8], the operation of a mechanical ventilator during inspiration $(0.9 \mathrm{~s})$ was modelled by means of a pressure generator with high internal resistance. A zero-flow pause of $0.1 \mathrm{~s}$ was introduced before a passive expiratory phase of $3 \mathrm{~s}$. A tidal volume of about $570 \mathrm{ml}$ was used without external positive end-expiratory pressure.

\subsection{NEP technique}

NEP technique consists of applying a sub-atmospheric pressure at the mouth during a tidal expiration and comparing the ensuing expiratory flow-volume curve with that of the previous expiration under steady state conditions [3]. NEP is usually applied to the expiratory circuit in early expiration and maintained for the rest of the expiration $[3,9]$. EFL occurs when the NEP-induced expiratory mouth flow equals the control expiratory flow.

\subsection{Numerical simulator}

The respiratory model, the artificial ventilator and the NEP technique were implemented numerically using Matlab-Simulink software. Simulink offers advantages with respect to other computer packages and related tools for 
nonlinear model design and simulation, albeit at the price of a considerable increase in computational time. It allows rapid prototyping and code generation for embedded systems, functional subsystem organisation, easy control and display of information, a helpful graphical interface for monitoring simulation, zooming and selecting areas of interest, and dialogue masks for setting and changing simulation parameters. The symmetrical lung description allowed the model to be represented mathematically by as few as 38 differential equations, reducing the simulation time of $8-10$ breaths, on a latest generation personal computer, to a few minutes.

\subsection{Simulation setting}

Normal respiratory conditions of artificial ventilation were simulated using model parameter values from the literature [6]. Branch lengths and diameters were taken as those measured by Weibel using resin casts of human lungs [5]. Compliances of the respiratory zone and chest wall were both set at $0.1 \mathrm{l} / \mathrm{cmH}_{2} \mathrm{O}$ $[10,11]$. A respiratory-zone (deep) resistance of $4 \mathrm{cmH}_{2} \mathrm{O} / 1 \mathrm{~s}$ was chosen [8].

To simulate a strong EFL condition we modified model parameters to reflect chronic obstructive pulmonary disease (COPD), which when severe, reveals expiratory flow limitation during mechanical ventilation. COPD is characterised by high airflow resistance determined by different possible causes: airway lumen reduction due to excessive secretions; thickening of airway walls, decreasing airway section; partial destruction of lung parenchyma producing weakening of radial traction and consequent airway narrowing.

In a trial-and-error computer procedure, observing the flow-volume curve after and before NEP application, we endeavoured to obtain high EFL by simulating severe COPD. In particular we simulated lumen reduction and/or wall thickening by decreasing airway diameters progressively (up to $50 \%$ ), from generation 5 downwards (small bronchi); we increased lower resistance by quintupling respiratory zone resistance; we increased loss of radial traction (facilitating airway collapse) by modifying airway elastic characteristics so as to have greater compliance than in normal conditions, at lower (sometimes negative) expiratory transmural pressures. The latter effect was obtained by decreasing the parameters $A_{2 n}$ by a factor of 3 to 6 with respect to their reference values, from generation 4 downward (segmental bronchi). As shown in eqn (4), parameters $A_{2 n}$ act on lower-left part of airway elastic characteristic (see also Fig. 3).

\section{Results}

Figure 4 shows the flow-volume loops of NEP-induced breath (continuous line) and previous control breath (dashed line) for normal and COPD computer simulated cases, applying a NEP of $-5 \mathrm{cmH}_{2} \mathrm{O}$ about $40 \mathrm{~ms}$ after the expiratory flow peak. Negative flows indicate expiratory phase. EFL was demonstrated in COPD by the fact that after a short initial flow spike, the expiratory flow-volume 
curve was practically superimposed on the control breath curve, unlike in the normal case. The concave shape of the flow-volume curve for simulated COPD is in line with experimental curves from mechanically ventilated patients studied by Valta et al. [3].
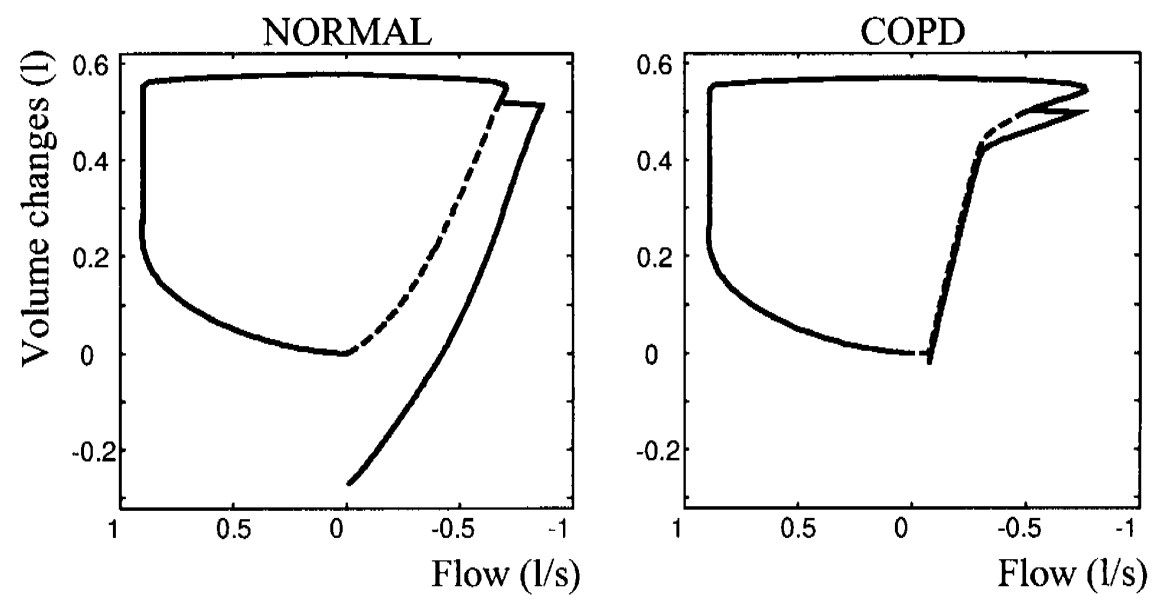

Figure 4: Flow-volume loops of test breath (continuous lines) and preceding control breath (dashed lines) obtained by the NEP technique.

Analysis of the expiratory time course of airway transmural pressures, $P_{t m}$, is useful for interpreting differences between the two simulated cases. Figure 5 shows $P_{t m}$ of lobar bronchi (continuous lines) and terminal bronchioles (dashed lines), during the control breath.

In the normal case, we observe that the $P_{t m}$ of the two generations considered, i.e. generations 2 and 16, decrease progressively during expiration, without reaching negative values, and their plots are almost superimposed. On the contrary, in the COPD case, the $P_{t m}$ of lobar bronchi behaves quite differently from that of terminal bronchioles. Soon after the start of expiration, it undergoes a sudden decrease in both generations and reaches very negative values in lobar bronchi. In this last generation, unlike in the normal case, $P_{t m}$ rises during expiration but remains negative, which suggests considerable bronchial compression in early expiration, tapering slightly at the end of expiration. In terminal bronchioles, on the other hand, $P_{t m}$ decreases, albeit slightly, throughout expiration. Finally, $P_{t m}$ shows much wider variations in the COPD than the normal case. 

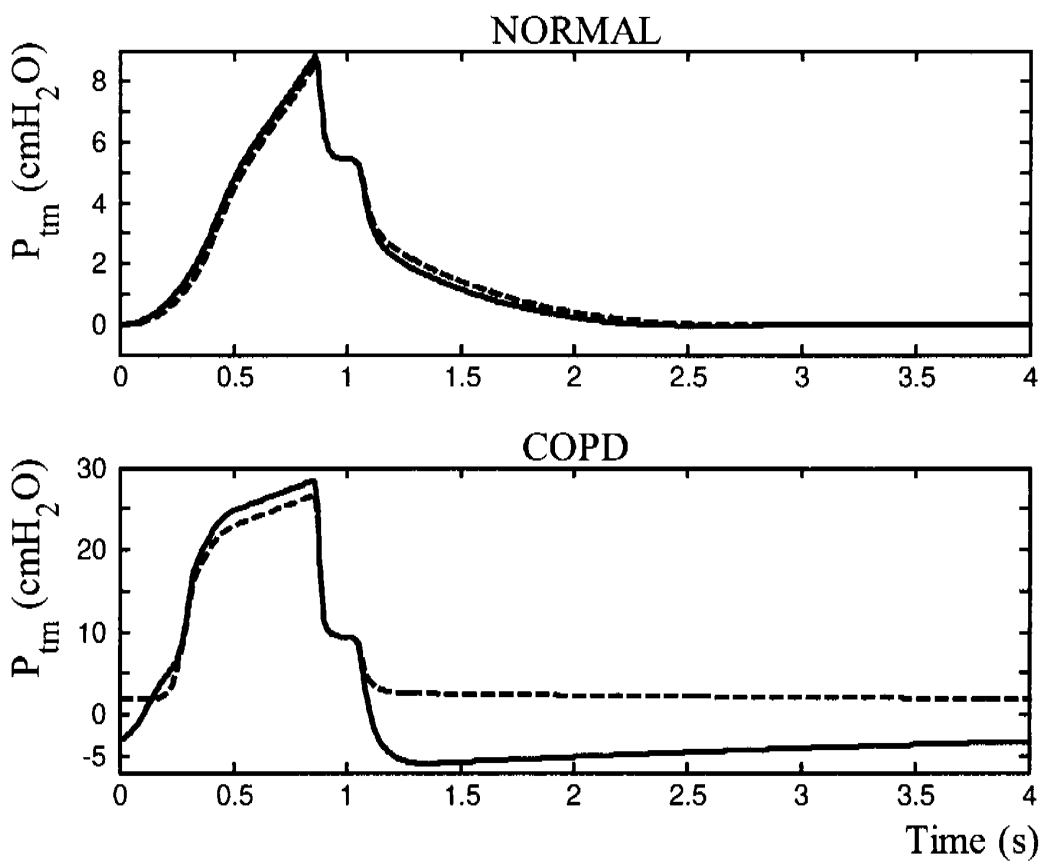

Figure 5: Airway transmural pressure, $P_{t m}$, of lobar bronchi (generation 2, continuous lines) and terminal bronchioles (generation 16, dashed lines), during control breath.

Figure 6 shows the generation diameters as percentage of their nominal values for normal and COPD cases. On the basis of the considerations about $P_{t m}$ in Fig. 5, it summarised the time variations in airway diameters during expiration, giving their percentages soon after the sudden drop in $P_{t m}$ (continuous lines) and at the end of expiration (dashed lines).

A marked reduction in segmental bronchial diameter can be observed in Fig. 6 for simulated EFL during expiration, down to about $19 \%$ of its maximum value, whereas the normal case has a diameter reduction down to about $67 \%$ for segmental bronchi (generation 4) at the end of expiration. Diameter changes during expiration in the COPD case differed from the normal case most radically in the first five generations where the diameter at the start of expiration was less than (or almost equal to) end-expiration diameter. On the other hand, from the small bronchi to bronchioles, diameter values reached a minimum at the end of expiration.

The normal case had a systematically larger diameter decrease during expiration for all generations. From generation 5 to terminal bronchioles, all the generation diameters varied from about $95 \%$ (early expiration) to about $74 \%$ (end of expiration) of their nominal values. 

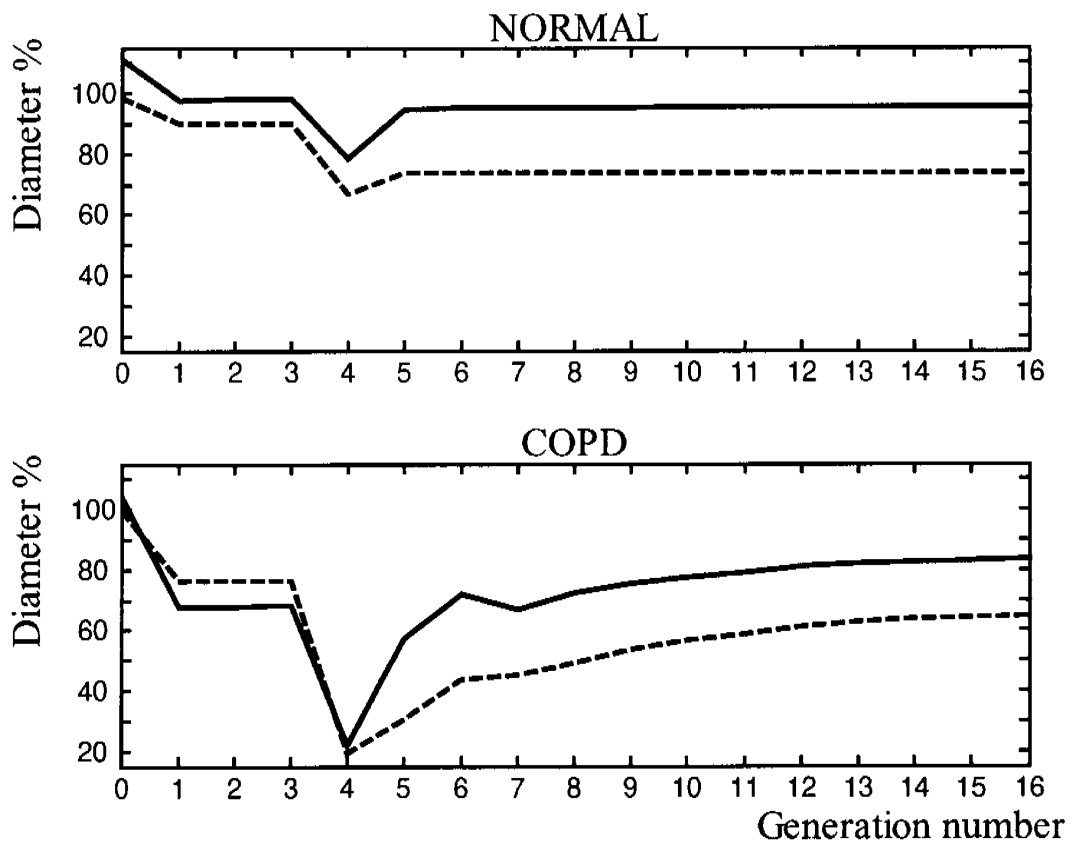

Figure 6: Conductive airway generation diameters as a percentage of their nominal values at the beginning (continuous lines) and end (dashed lines) of expiration.

\section{Conclusions}

The results obtained using the nonlinear model of breathing mechanics to simulate a normal respiratory case and a severe COPD case in artificial ventilation, and the corresponding flow-volume curves, in line with experimental data from Valta et al. [3], made it possible to interpret the EFL phenomenon. It originates from a rise in respiratory resistances and a reduction in rigidity of the conductive airways, evident in the following two distinct phases.

In the first part of expiration (high alveolar pressures), the airways closest to trachea (generations 1-4) narrow significantly, because of their increased pathological collapsability (higher compliance), greatly limiting outflow. Upper airway compression is aggravated by lower transmural pressures due to respiratory resistances, augmented by obstructions which produce a greater pressure drop from the alveoli to the upper airways.

During the second part of expiration, as alveolar pressure gradually reduces, the compliant lower generations are increasingly involved in the diameter reduction. This causes a noteworthy rise in their resistance and consequently a marked limitation of flow rate, which partly re-inflates the upper airways, increasing their diameters. 


\section{Acknowledgements}

This work was supported in part by the Italian Ministry of Education, University and Research.

\section{References}

[1] Armaganidis, A., Stavrakaki-Kallergi, K., Koutsoukou, A. Lymberis, A., Milic-Emili, J. \& Roussos, C., Intrinsic positive end-expiratory pressure in mechanically ventilated patients with and without tidal expiratory flow limitation. Critical Care Medicine, 28, pp. 3837-3842, 2000.

[2] Koulouris, N.G., Dimopoulou, I., Valta, P., Finkelstein, R., Cosio, M.G. \& Milic-Emili, J., Detection of expiratory flow limitation during exercise in COPD patients. Journal of Applied Physiology, 82, pp. 723-731, 1997.

[3] Valta, P., Corbeil, C., Lavoie, A., Campodonico, R., Koulouris, N, Chasse, M., Braidy, J. \& Milic-Emili, J., Detection of expiratory flow limitation during mechanical ventilation. American Journal of Respiratory and Critical Care Medicine, 150, pp. 1311-1317, 1994.

[4] Bernardi, F., Cevenini, G., Massai, M.R. \& Barbini, P., A symmetrical computer model of nonlinear mechanical properties of the tracheobronchial airways. Proc. of the $9^{\text {th }}$ Mediterranean Conference on Medical Biological Engineering and Computing, pp. 911-914, Pula, June 2001.

[5] Weibel, E.R., Morphometry of the Human Lung, Springer-Verlag: Berlin, 1963.

[6] Cevenini, G., Bernardi, F., Massai, M.R. \& Barbini, P., A morphometric interpretation of conductive airway compliance in mechanical ventilation. Proc. of the $5^{\text {th }}$ Int. Symp. on Computer Methods in Biomechanics and Biomedical Engineering, Rome, November 2001.

[7] Pardaens, J., Van de Woestijne, K.P. \& Clément, J., A physical model of expiration. Journal of Applied Physiology, 33, pp. 479-490, 1972.

[8] Barbini, P., Bernardi, F., Cevenini, G., Massai, M.R., Avanzolini, G. \& Gnudi, G., Effect of compliant intermediate airways on total respiratory resistance and elastance in mechanical ventilation. Medical Engineering \& Physics, 23, pp. 185-194, 2001.

[9] Johnson, B.D., Beck, K.C., Zeballos, R.J. \& Weisman, I.M., Advances in pulmonary laboratory testing. Chest, 116, pp. 1377-1387, 1999.

[10] West, J.B., Pulmonary Pathophysiology, The Essentials, Williams \& Wilkins, Baltimore, $4^{\text {th }}$ ed., 1992.

[11] Nunn, J.F., Applied Respiratory Physiology, Butterworths, London, $3^{\text {rd }}$ ed., 1987. 